\title{
A comparison of existing co-ordinate transformation models and parameters in Australia
}

\author{
W. E. Featherstone \\ School of Surveying and Land Information \\ Curtin University of Technology \\ Perth WA 6001
}

\begin{abstract}
Four standard procedures to transform curvilinear co-ordinates from the Australian Geodetic Datum 1984 to the World Geodetic System 1984 are compared. These comprise the Bursa-Wolf model with the national set of seven parameters currently used by Federal and State surveying and mapping authorities, the standard Molodensky model with the five parameters used by the United States Defense Mapping Agency, the simple three-parameter model with the origin shifts taken from the Bursa-Wolf and standard Molodensky models, and the multiple regression equations as determined by the Defense Mapping Agency. The differences between the resulting co-ordinates can reach 4.2 metres over continental Australia, which has implications for the final approach adopted to transform to the Geocentric Datum of Australia. The arguments are presented in favour of more suitable transformation strategies using projective transformation models, which are able to simultaneously correct any known errors existing in the Australian Geodetic Datum. These models also allow the direct transformation of both Australian Geodetic Datum 1966 and Australian Geodetic Datum 1984 coordinates in a single procedure, which will be of benefit to those States which rely upon older geodetic datums.
\end{abstract}

\section{INTRODUCTION}

Australia's transition to the use of the Geocentric Datum of Australia (GDA) for surveying and mapping by the 1st January, 2000 will require that existing spatial data are transformed to this new co-ordinate datum (Featherstone, 1994 and 1996; Steed, 1995; Inter-governmental Committee on Surveying and Mapping, 1994; Higgins, 1994; Manning and Harvey, 1994). Featherstone (1994 and 1996), among other authors, only presents the seven-parameter conformal transformation model using the Higgins (1987) constants. However, there are several alternative transformation models and parameters currently available for Australia which can also be employed for this purpose.

This paper compares four mathematical models and their associated parameters for the transformation of curvilinear co-ordinates from the Australian Geodetic Datum 1984 (AGD84) to the World Geodetic System 1984 (WGS84), which can be assumed compatible with the GDA for many practical purposes. These are:

1. A seven-parameter conformal transformation using the Bursa-Wolf model (Bursa, 1962; Wolf, 1963), where the transformation is staged via Cartesian co-ordinates and uses the parameters of Higgins (1987).

2. A five-parameter conformal transformation based on a curvilinear version of the Molodensky-Badekas model (Molodensky et al., 1962; Badekas, 1969) with the Defense Mapping Agency's (1991) parameters.

3. The multiple regression equation approach of the Defense Mapping Agency (1991), which is a spatially varying or projective transformation. (The latter two approaches are designed to operate directly on the curvilinear co-ordinates, and thus provide a conceptually more direct transformation.) 
4. A three-parameter transformation model using only the origin shifts of the Higgins and Defense Mapping Agency parameters, which is theoretically inappropriate but provides an interesting comparison.

The merits of conformal transformation models in Australia has previously been discussed by Burford (1985) and Harvey (1986). Both authors agree that the Molodensky-Badekas model is more suited to the integration of satellite-derived co-ordinates with terrestrial co-ordinates, as is predicted by the theory. Burford also shows that regional seven-parameter approaches are more appropriate than a single national model in Australia, and this is corroborated by Harvey, provided that the differences between parameters are statistically significant.

This paper builds upon these findings to some extent, but is more concerned with the practical transformation of terrestrial AGD84 co-ordinates to the WGS84 system and their numerical differences when using published transformation parameters. A series of recommendations is given for the future determination of transformation parameters from the AGD to the GDA, where a projective transformation should be used in preference to a single, national conformal model. When using the latter approach, errors that may exist in the AGD are simply transformed, albeit to a lesser extent, to the GDA, thus neglecting some of the benefits that can be achieved as a by-product of this change of datum.

\section{The geometry of the problem}

Although only the transformation of the curvilinear latitude and longitude is required in the case of the GDA, the transformation is often achieved using a three-dimensional approach. Figure 1 shows a cross-section of the geometry of this transformation, where curvilinear co-ordinates of a point on or near the Earth's surface can be expressed with respect to any number of reference surfaces. Therefore, the common [mis]conception that latitude and longitude are unique is not strictly true. Instead, they also depend upon the spheroidal reference surface and co-ordinate datum being utilised. Only by providing this auxiliary information are the latitude and longitude truly unique.

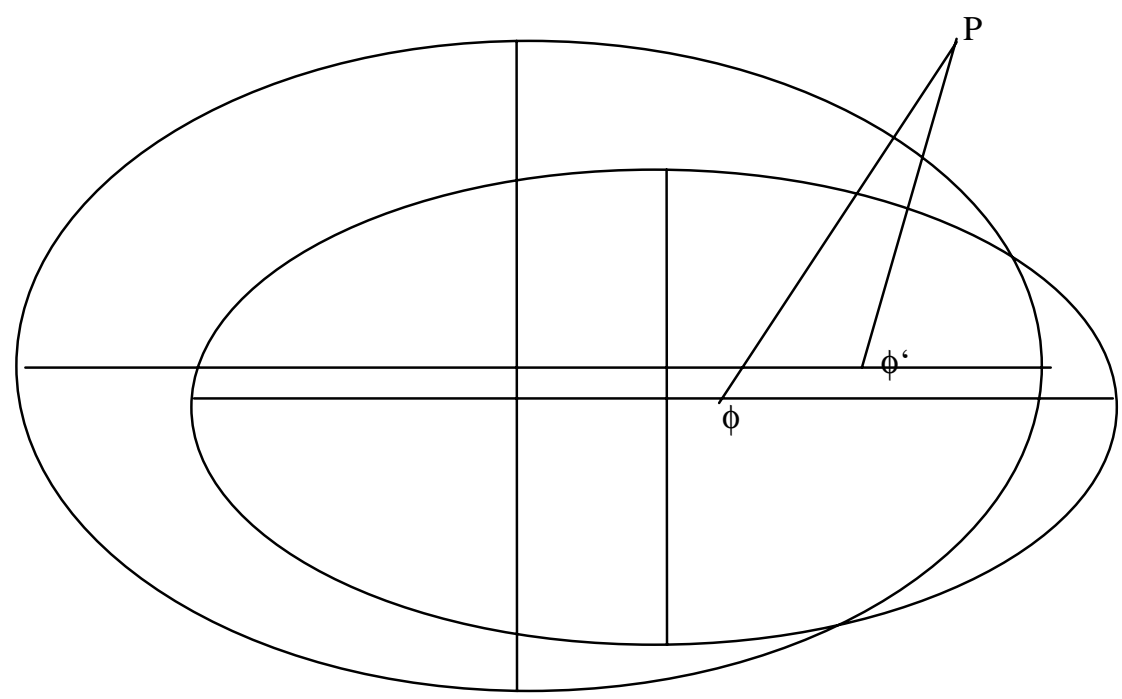

Figure 1. A cross-section of two reference spheroids: The fixed point $P$ in three-dimensional space has different curvilinear co-ordinates by virtue of the reference surface (and co-ordinate datum) chosen. The latitude $\phi$ refers to the surface of the smaller reference spheroid, whereas the latitude $\phi$ ' refers to the larger reference spheroid. 


\section{THE CARTESIAN TRANSFORMATIONS}

When taking the Cartesian approach to this co-ordinate transformation, the curvilinear co-ordinates must first be converted to Cartesian co-ordinates centred on the particular reference spheroid which is associated with the initial datum (eg. the Australian National Spheroid, or ANS, in the case of the AGD84). These Cartesian coordinates are then transformed to Cartesian co-ordinates centred on the spheroid associated with the desired datum (eg. the WGS84 or the GDA) using one of many conformal models. Finally, these transformed Cartesian co-ordinates are converted to curvilinear co-ordinates on the new datum and spheroid.

\section{Bowring's algorithm}

The forward conversion from curvilinear co-ordinates $(\phi, \lambda, h)$ to Cartesian co-ordinates $(X, Y, Z)$ is relatively straightforward, and is given by

$$
\begin{aligned}
& X=(v+h) \cos \phi \cos \lambda \\
& Y=(v+h) \cos \phi \sin \lambda \\
& Z=\left[v\left(1-e^{2}\right)+h\right] \sin \phi
\end{aligned}
$$

where the radius of curvature of the prime vertical $(v)$ is

$$
v=\mathrm{a}\left(1-\mathrm{e}^{2} \sin ^{2} \phi\right)^{-1 / 2}
$$

and $a$ and $e$ are the semi-major-axis length and first numerical eccentricity, respectively, of the reference spheroid associated with the initial datum (ANS in this case).

For most practical purposes it is not usually necessary to use the spheroidal height $(h)$ in equations (1), (2) and (3) because the forward (and reverse) conversion is only weakly dependent upon height. For instance, the ANS spheroidal heights on land in Australia comprise less than 0.05 percent of the radius of the prime vertical, and thus only affect geographical co-ordinates by nine centimetres at most (Featherstone, 1994). Therefore, the third dimension will be omitted from these comparisons. This omission is also justified by the fact that the change to the GDA will not affect existing Australian Height Datum elevations.

The reverse transformation from Cartesian to curvilinear co-ordinates is more involved because the direct inversion of equations (1), (2) and (3) requires some iteration for the solution of latitude (Bowring, 1985). Closed (non-iterative) solutions to this problem do exist by using quartic equations (eg. Paul, 1973; Borkowski, 1989), but this discussion is not concerned with the relative merits of closed and iterative formulae, and Bowring's algorithm will be used for all subsequent computations; this is

$$
\phi=\arctan \frac{Z+\varepsilon^{2} b \sin ^{3} u}{\sqrt{X^{2}+Y^{2}}-e^{2} a \cos ^{3} u}
$$

where $b$ and $\varepsilon$ are the semi-minor-axis length and the second numerical eccentricity, respectively, of the reference spheroid associated with the new datum, and $u$ is the parametric latitude, given by

$$
\mathrm{u}=\arctan \left(\frac{\mathrm{b}}{\mathrm{a}} \tan \phi\right)
$$

The initial estimate of the parametric latitude can either use the AGD84 latitude of the point to be transformed, or Bowring's equation

$$
u=\arctan \frac{b Z}{a \cdot \sqrt{X^{2}+Y^{2}}}\left(1+\frac{\varepsilon^{2} b}{\sqrt{X^{2}+Y^{2}+Z^{2}}}\right)
$$


The longitude is given by the simple, closed trigonometric expression

$$
\lambda=\arctan \frac{Y}{X}
$$

Once the curvilinear co-ordinates have been converted to their Cartesian counterparts using equations (1), (2) and (3), the following three mathematical models can be used to transform these Cartesian co-ordinates between the respective datums.

\section{The Bursa-Wolf transformation model}

The Bursa-Wolf (Bursa, 1962; Wolf, 1963) seven-parameter conformal model for transforming threedimensional Cartesian co-ordinates between datums is especially suited to satellite datums on a global scale (Krakiwsky and Thomson, 1974). This comprises an origin shift from the geocentre in three-dimensional space $\left(X_{0}, Y_{0}, Z_{0}\right)$, rotation of the vector position $(r x, r y, r z)$, and a scale change $(d s)$. These are applied in matrix-vector form via

$$
\left[\begin{array}{l}
X \\
Y \\
Z
\end{array}\right]_{W}=\left[\begin{array}{l}
X_{0} \\
Y_{0} \\
Z_{0}
\end{array}\right]+\left[\begin{array}{ccc}
1+d s & r Z & -r y \\
-r Z & 1+d s & r X \\
r y & -r X & 1+d s
\end{array}\right]\left[\begin{array}{l}
X \\
Y \\
Z
\end{array}\right]_{A}
$$

where the subscripts $W$ and $A$ refer to the WGS84 and AGD84 geodetic datums, respectively. The single threeby-three rotation matrix is simplified from three separate rotation matrices by assuming that each axial rotation is differentially small (typically less than five arc seconds for most geodetic networks), thus permitting binomial series expansions of the sine and cosine terms for radian measure. The numerical values of seven parameters required for this Bursa-Wolf transformation model in Australia have been determined by Higgins (1987), and are summarised in Table 1.

\begin{tabular}{|c|c|c|}
\hline parameter & value & units \\
\hline$\Delta X$ & -116.00 & metres \\
\hline$\Delta Y$ & -50.47 & metres \\
\hline$\Delta Z$ & 141.69 & metres \\
\hline$r x$ & -0.230 & arc seconds \\
\hline$r y$ & -0.390 & arc seconds \\
\hline$r z$ & -0.344 & arc seconds \\
\hline$d s$ & $0.0983 \times 10^{-6}$ & -- \\
\hline
\end{tabular}

Table 1. The Bursa-Wolf transformation parameters from AGD84 to WGS84 (after Higgins, 1987)

\section{The Molodensky-Badekas model}

The Molodensky-Badekas model (Molodensky et al., 1962; Badekas, 1969) is also a seven-parameter conformal transformation of three-dimensional Cartesian co-ordinates between datums, but is more suited to the transformation between terrestrial and satellite datums (Krakiwsky and Thomson, 1974). It is worth pointing out some discrepancies in the exact citation of this approach. Soler $(1976$, p.2) states ... the differential equations published in the English translation of Molodensky et al. are equivalent to conventional conformal transformations. This dissipates the confusion created recently by some authors (Badekas, 1969; Krakiwsky and Thomson, 1974), who credited Molodensky et al. with a model they never wrote. However, Krakiwsky and 
Thomson (1974, p.610) acknowledge that the original Molodensky model is different to that credited to them. Nevertheless, this paper will continue to use the confused citation of Molodensky-Badekas as this has been employed in earlier Australian literature on this subject.

The Molodensky-Badekas model, as given by Krakiwsky and Thomson (1974), Burford (1985) and Harvey (1986), is

$$
\left[\begin{array}{l}
X \\
Y \\
Z
\end{array}\right]_{W}=\left[\begin{array}{c}
\Delta X \\
\Delta Y \\
\Delta Z
\end{array}\right]+\left[\begin{array}{l}
X_{m} \\
Y_{m} \\
Z_{m}
\end{array}\right]+\left[\begin{array}{ccc}
1+d s & r Z & -r y \\
-r Z & 1+d s & r X \\
r y & -r X & 1+d s
\end{array}\right]\left[\begin{array}{l}
X_{A}-X_{m} \\
Y_{A}-Y_{m} \\
Z_{A}-Z_{m}
\end{array}\right]
$$

where $(\Delta X, \Delta Y, \Delta Z)$ are the shifts between the barycentre or centroid of the terrestrial network $\left(X_{m}, Y_{m}, Z_{m}\right)$ and the rotation matrix and scale change are theoretically identical to the Bursa-Wolf model. Therefore, the only conceptual difference between the Molodensky-Badekas and Bursa-Wolf models is the choice of the point about which the axial rotations and scale change are applied. As this point is the barycentre for the MolodenskyBadekas model, this model offers a more appropriate option for the transformation between terrestrial and satellite datums. Theoretically, the Bursa-Wolf and Molodensky-Badekas models should give the same results when the same data are used to determine the respective sets of transformation parameters. However, it is not possible to test this here because the Molodensky-Badekas parameters are not currently available in Australia. It is possible to derive them from the Bursa-Wolf parameters, but the co-ordinates of the barycentre would also need to be known, which is not the case (Harvey, 1986).

\section{The three-parameter model}

This model simply applies a three-dimensional origin shift, with little regard for any scale changes or rotations. Therefore, it is coarse, but also extremely simple to implement. The Cartesian co-ordinates from the initial datum are simply added to the origin shift, then converted to curvilinear co-ordinates on the new datum. In vector form, this reads

$$
\begin{aligned}
& {\left[\begin{array}{l}
X \\
Y \\
Z
\end{array}\right]_{W}=\left[\begin{array}{c}
X_{0} \\
Y_{0} \\
Z_{0}
\end{array}\right]+\left[\begin{array}{l}
X \\
Y \\
Z
\end{array}\right]_{A}} \\
& {\left[\begin{array}{l}
X \\
Y \\
Z
\end{array}\right]_{W}=\left[\begin{array}{l}
\Delta X \\
\Delta Y \\
\Delta Z
\end{array}\right]+\left[\begin{array}{l}
X \\
Y \\
Z
\end{array}\right]_{A}}
\end{aligned}
$$

where the origin shifts can be taken from the Bursa-Wolf model in equation (11a) or the Molodensky-Badekas model in equation (11b). However, the different correlations between rotations and shifts in each model (Burford, 1985; Harvey, 1986) suggest that the numerical results may differ, and depend upon the original determination of the origin shifts. This will be demonstrated later.

\section{THE CURVILINEAR TRANSFORMATIONS}

The following discussion will consider the curvilinear form because the associated parameters are readily available in Defense Mapping Agency (1991). Also, the curvilinear transformations are conceptually more simple as they directly produce a co-ordinate change in degrees instead of converting via Cartesian co-ordinates. 


\section{The Standard Molodensky model}

This model is applied in curvilinear form by using equations (1), (2), (3) and the Jacobian matrix for two different ellipsoids (cf. Vanicek and Krakiwsky, 1986 p.333) in equation (10), and where the Cartesian origin shifts are embedded in equations (12) and (13). However, the standard Molodensky transformation as used by the Defense Mapping Agency is only a five-parameter version of the complete Molodensky-Badekas model. It simply applies a three-dimensional origin shift from the geocentre $(\Delta X, \Delta Y, \Delta Z)$ in conjunction with a scale change provided by the difference in semi-major-axis length $(\Delta a)$ and flattening $(\Delta f)$ of the respective reference spheroids.

From Defense Mapping Agency (1991), the standard Molodensky transformation is given in curvilinear form by

$$
\begin{aligned}
& \phi_{W}=\phi_{A}+[-\Delta X \sin \phi \cos \lambda+\Delta Y \sin \phi \sin \lambda+ \\
& +\Delta Z \cos \phi]+\frac{\Delta a}{a}\left(v e^{2} \sin \phi \cos \phi\right)+ \\
& \left.+\Delta f\left(\frac{\rho a}{b}+\frac{v b}{a}\right) \sin \phi \cos \phi\right] \cdot[v+h]^{-1} \\
& \lambda_{W}=\lambda_{A}+\frac{-\Delta X \sin \lambda+\Delta Y \cos \lambda}{(v+h) \cos \phi}
\end{aligned}
$$

where the radius of curvature of the meridian $(\rho)$ is

$$
\rho=\frac{a\left(1-e^{2}\right)}{\left(1-e^{2} \sin ^{2} \phi\right)^{3 / 2}}
$$

and the numerical values of $a$ and $e$ refer to the reference spheroid associated with the initial datum.

Equations (12) and (13) may appear daunting at first sight, but are no more complicated to implement than the Bursa-Wolf and Bowring algorithms, especially when they are treated as a five-by-five matrix equation. Computer subroutines, written in Sun FORTRAN77, for the standard Molodensky, Bursa-Wolf, and threeparameter models and the Bowring algorithms are available from the author.

The transformation parameters and other constants associated with the standard Molodensky model are shown in Table 2 and were taken from Defense Mapping Agency (1991), who used 90 Doppler stations across Australia for their determination.

\begin{tabular}{|c|c|c|}
\hline parameter & value & units \\
\hline$X_{0}$ & -134 & metres \\
\hline$Y_{0}$ & -48 & metres \\
\hline$Z_{0}$ & 149 & metres \\
\hline$\Delta a$ & -23 & metres \\
\hline$\Delta f$ & $-8.1204 \times 10^{-8}$ & -- \\
\hline$a$ & 6378160 & metres \\
\hline$f$ & $1 / 298.25$ & -- \\
\hline
\end{tabular}

Table 2. The standard Molodensky transformation parameters from AGD84 to WGS84 (after Defense Mapping Agency, 1991) 
As predicted by theory, the origin shift parameters used with the standard Molodensky transformation (Table 2) are different to the origin shifts used with the Bursa-Wolf transformation (Table 1) because the former refer to the barycentre of the terrestrial geodetic network. Moreover, the origin shift parameters in Table 2 are an artefact of the implementation of the standard Molodensky model by the Defense Mapping Agency, where the axial rotations are assumed to be zero (which is not strictly valid) and are thus absorbed into the origin shift terms, to a certain extent, because of the correlations between them (Burford, 1985; Harvey, 1986; Thomson and Krakiwsky, 1974).

\section{The Multiple Regression Equations}

The multiple regression equation approach to datum transformations (Applebaum, 1982) is both a curvilinear and a projective method. Theoretically, it is superior to the conformal transformation models and their derivatives because it can account for non-linear distortions between the terrestrial and satellite datums. These are usually suspected to reside in the terrestrial datum because satellite-based surveys are often superior to conventional terrestrial surveys, especially at continental scales.

In an ideal situation where no errors exist in either the terrestrial or satellite datum, the conformal models would offer a suitable and accurate approach. However, this is not usually the case, especially at continental scales, and a projective transformation provides a superior option to the linear conformal models (. The primary advantage of this projective method lies in the fact that localised distortions in the AGD can be modelled and accounted for, thus giving superior transformations to WGS84 or the GDA for all applications, whether they be geodetic or cartographic. Therefore, if sufficient points are available with co-ordinates known in both datums (as is the case in Australia), the projective transformation can be determined with some confidence.

The following projective, multiple regression equations given by the Defense Mapping Agency (1991) were determined for mainland Australia from 90 satellite-positioned stations co-located with AGD84 ground points. As with the conformal transformations, these represent a form of interpolation (Harvey, ibid.) and should not be used outside the area for which they were determined.

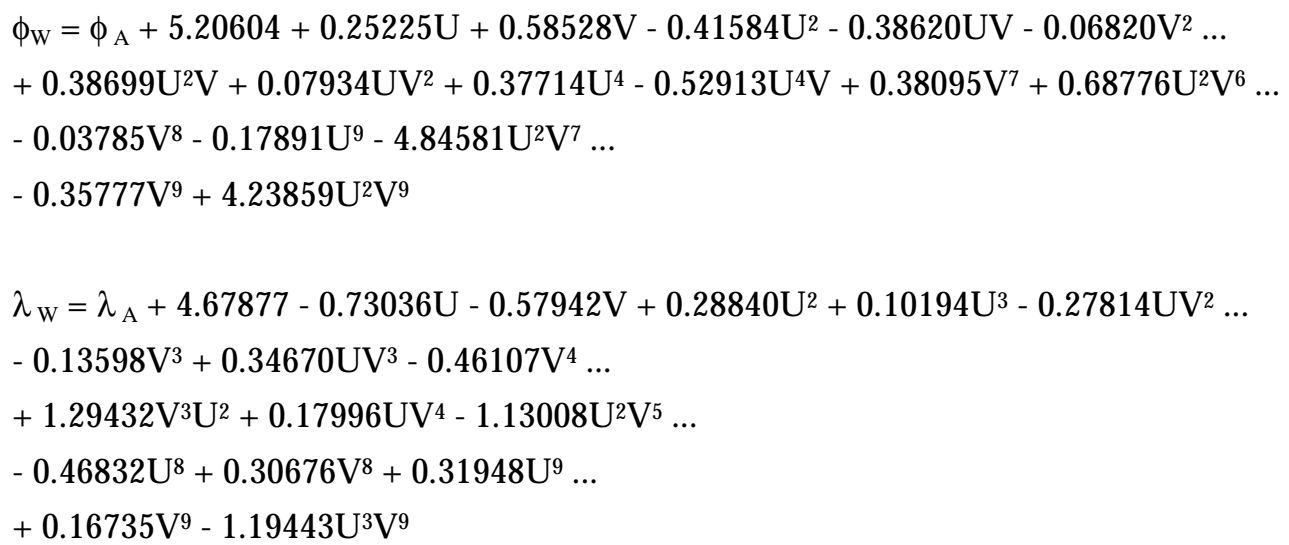

where $\mathrm{U}$ and $\mathrm{V}$ are the scaled and normalised latitude and longitude, respectively, and are given by

$$
\begin{aligned}
& \mathrm{U}=0.05235988\left(\phi_{\mathrm{A}}+27^{\circ}\right) \\
& \mathrm{V}=0.05235988\left(\lambda_{\mathrm{A}}-134^{\circ}\right)
\end{aligned}
$$




\section{THE NUMERICAL COMPARISONS}

In all the following comparisons, the Bursa-Wolf model using the Higgins (1987) parameters is used to provide the control data and the other four transformation models with their associated parameters were simply subtracted from this. This approach is chosen because the Bursa-Wolf/Higgins approach is currently the most commonly used method to transform between AGD84 and WGS84 co-ordinates in Australia. Table 3 summarises these combinations and the abbreviations which will be used in the following part of this paper.

\begin{tabular}{|c|c|}
\hline transformation models and parameters compared & abbreviation \\
\hline $\begin{array}{c}\text { Bursa-Wolf model (9) with Higgins parameters minus standard Molodensky model } \\
\text { (10) with Defense Mapping Agency parameters }\end{array}$ & BWH - MDMA \\
\hline $\begin{array}{c}\text { Bursa-Wolf model (9) with Higgins parameters minus three-parameter model with } \\
\text { Defense Mapping Agency parameters (11a) }\end{array}$ & BWH - TDMA \\
\hline $\begin{array}{c}\text { Bursa-Wolf model (9) with Higgins parameters minus three-parameter model with } \\
\text { Higgins parameters (11b) }\end{array}$ & BWH - TH \\
\hline $\begin{array}{c}\text { Bursa-Wolf model (9) with Higgins parameters minus multiple regression equations } \\
\text { with Defense Mapping Agency constants (16 and 17) }\end{array}$ & BWH - MREDMA \\
\hline
\end{tabular}

Table 3. A summary of the transformation models and parameters compared together with their abbreviations.

Geographical grids of the respective co-ordinate differences between the transformed WGS84 curvilinear coordinates were computed at a two-degree interval in the region $112^{\circ}<\lambda<154^{\circ}$ and $-44^{\circ}<\phi<-8^{\circ}$. These were then converted to distances in metres and azimuths in degrees by using a spherical Earth approximation. It is acknowledged that more exact co-ordinate differences should be computed with respect to the geodesic. However, the approximate conversion used here is sufficient to illustrate the relative differences between the transformation models and parameters. Figures 2, 3, 4 and 5 show the co-ordinate differences for the approaches listed in Table 3 as contour lines and two-dimensional vectors. 


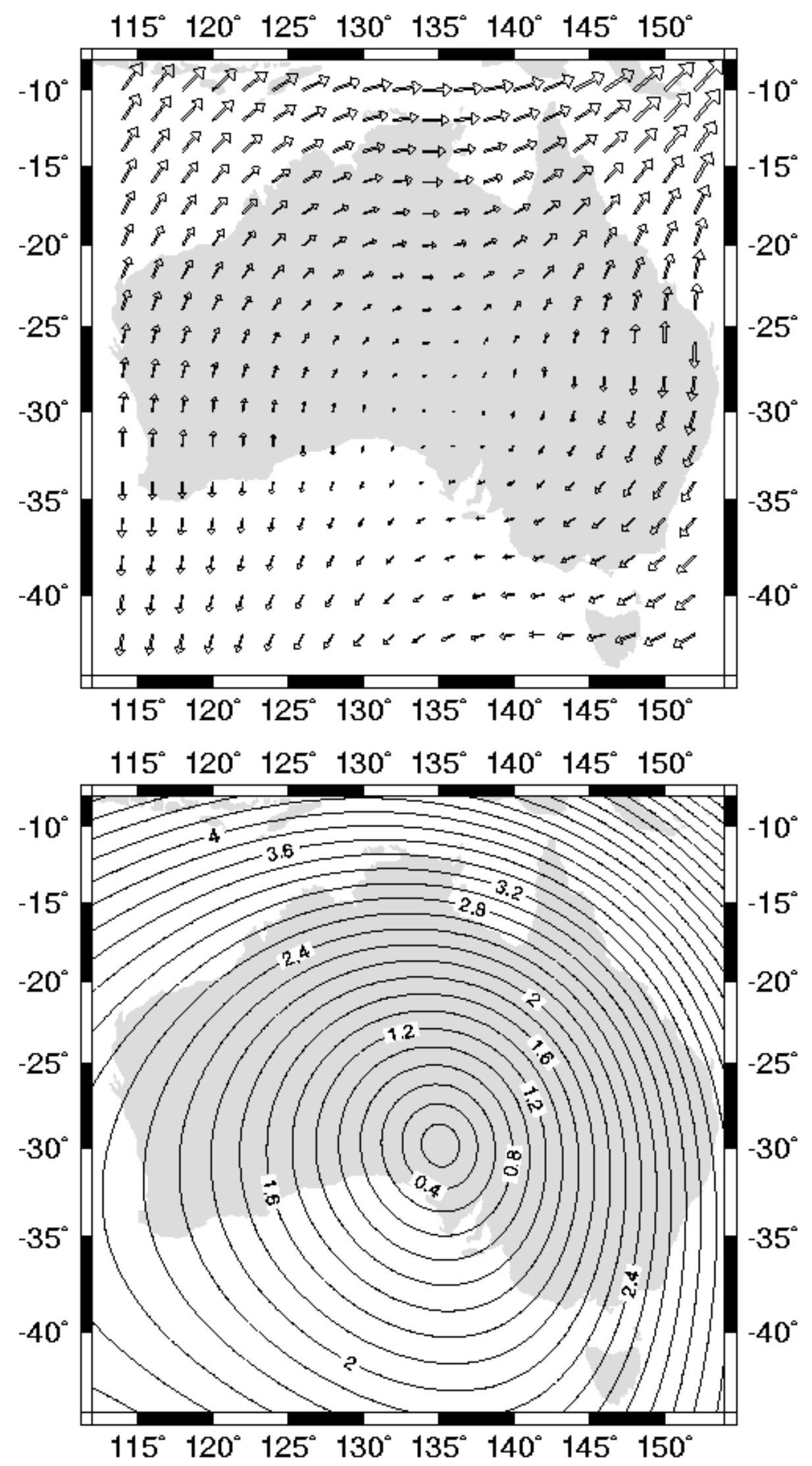

Figure 2. A contour map (a) and vectors (b) of the difference (in metres) between WGS84 co-ordinates derived from AGD84 co-ordinates using the BWH minus MDMA approaches. Mercator projection. 


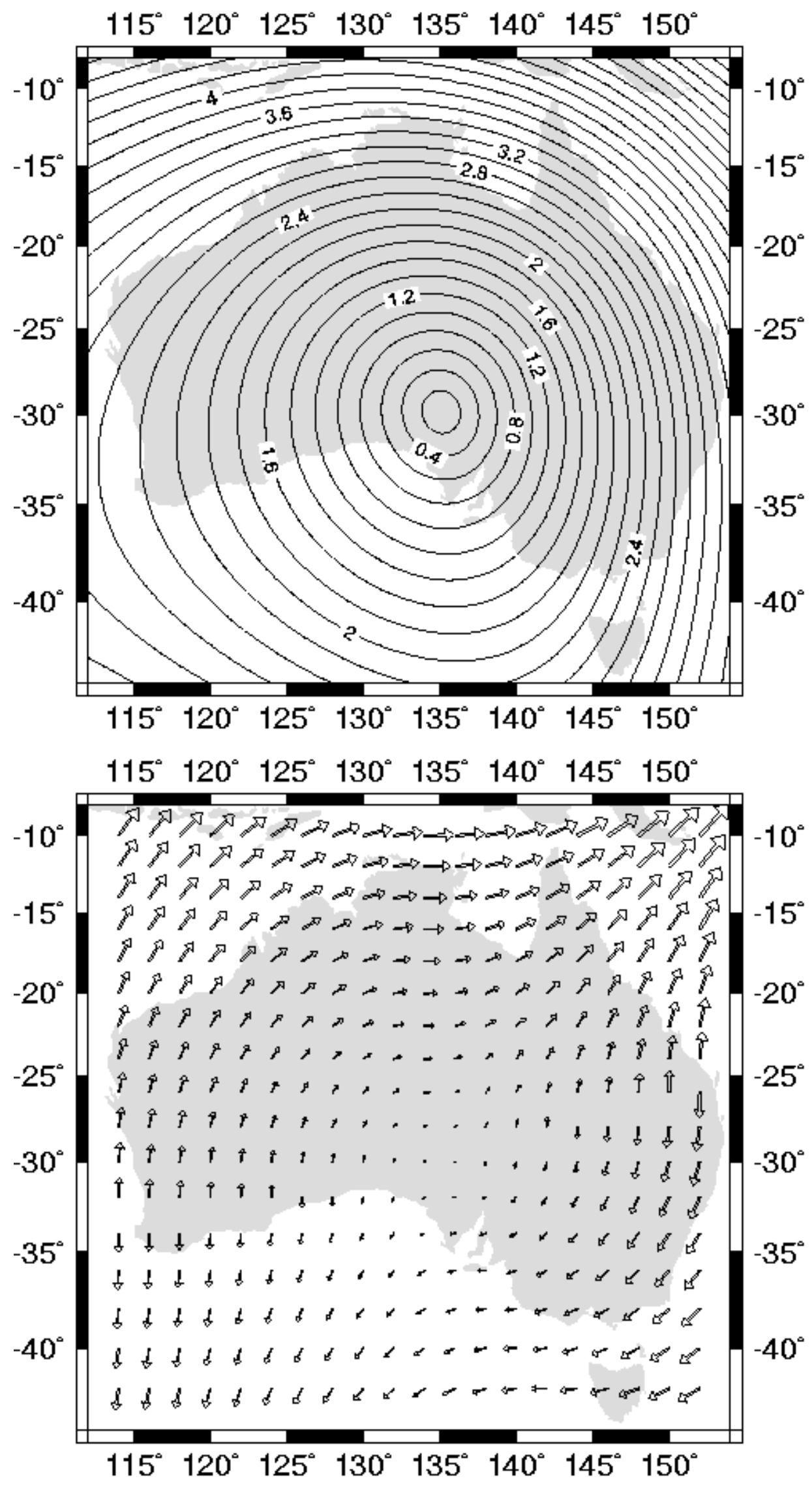

Figure 3. A contour map (a) and vectors ( $b$ ) of the difference (in metres) between WGS84 co-ordinates derived from AGD84 co-ordinates using the BWH minus TDMA approaches. Mercator projection. 


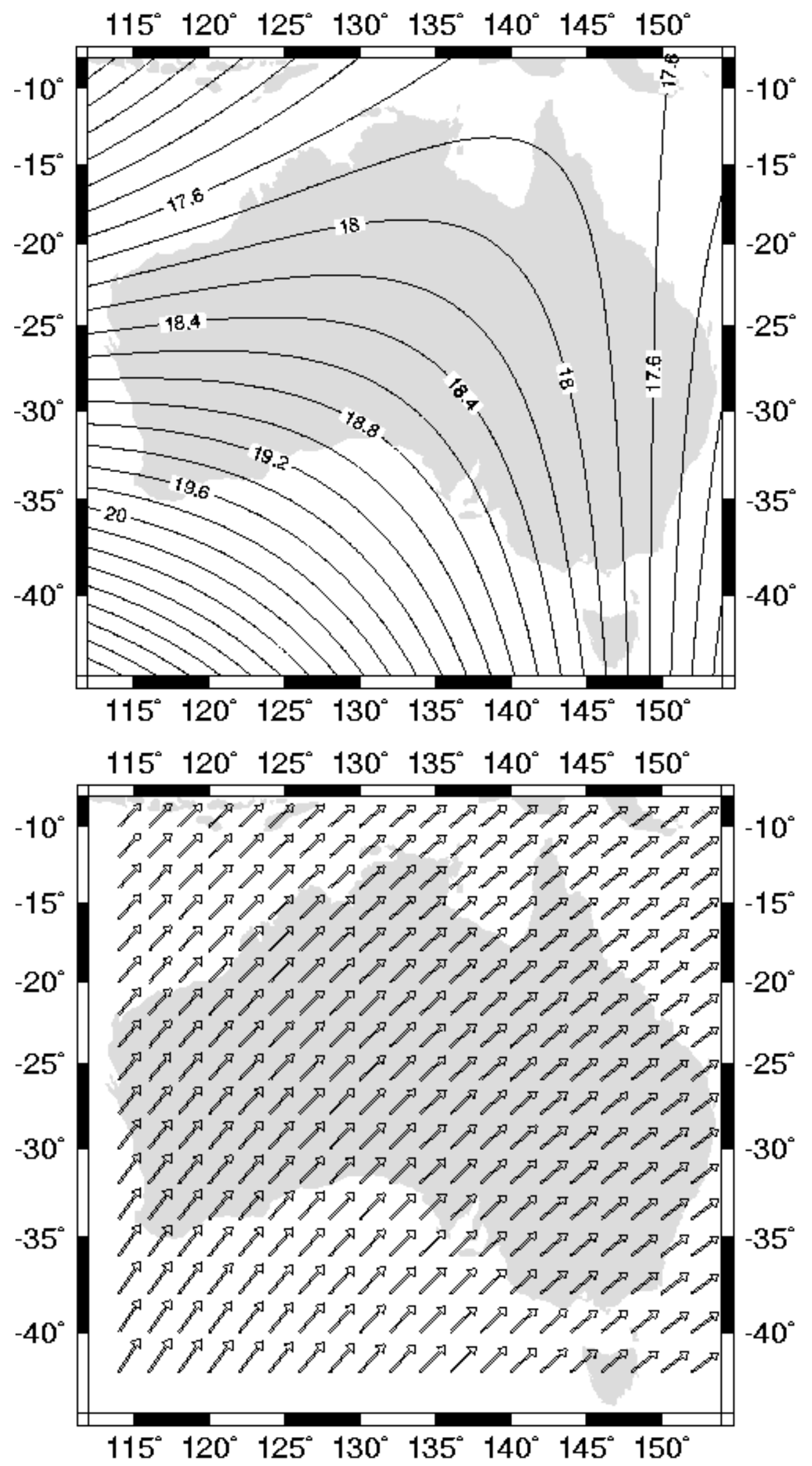

Figure 4. A contour map (a) and vectors (b) of the difference (in metres) between WGS84 co-ordinates derived from AGD84 co-ordinates using the BWH minus TH approaches. Mercator projection. 

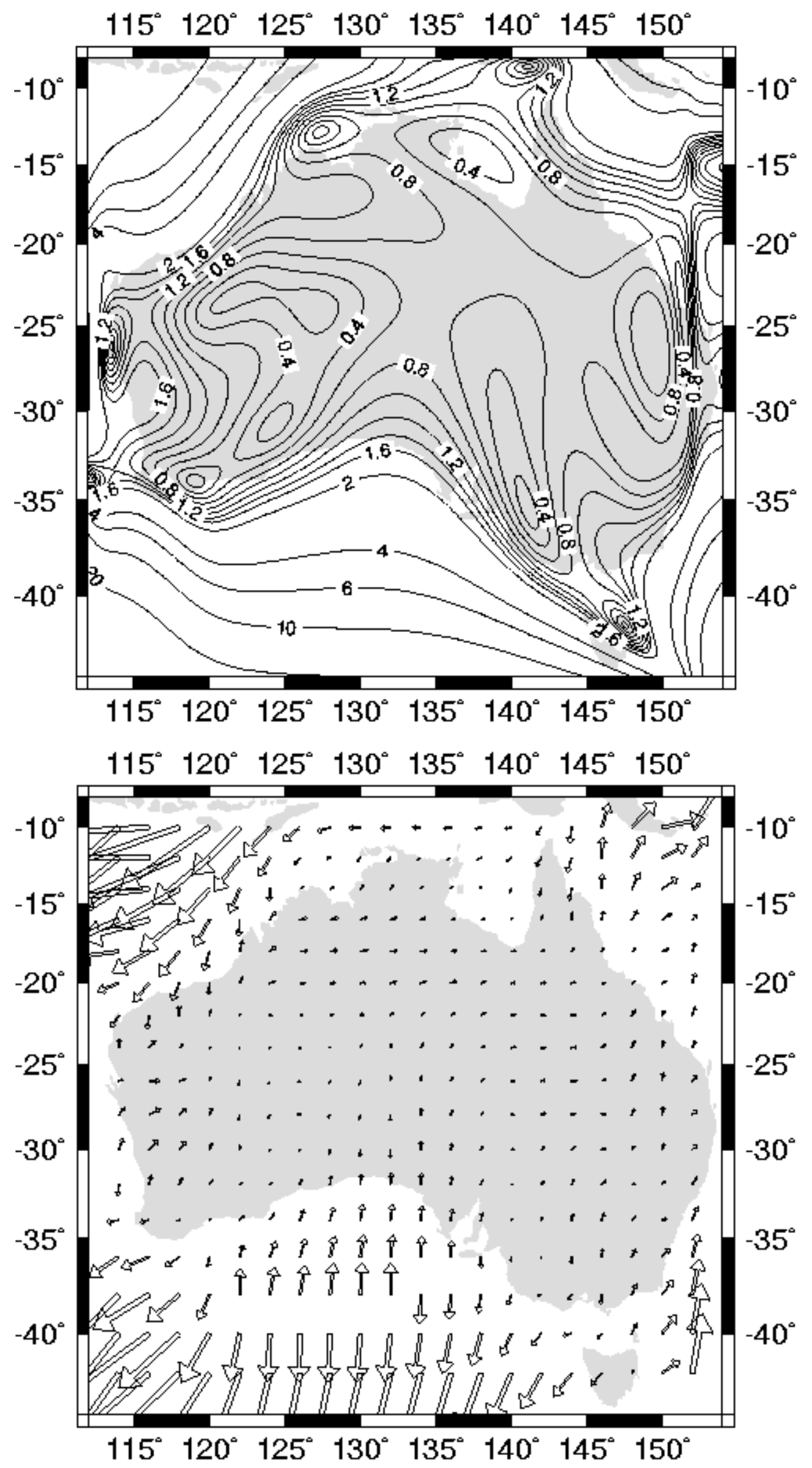

Figure 5. A contour map (a) and vectors $(b)$ of the difference (in metres) between WGS84 co-ordinates derived from AGD84 co-ordinates using the BWH minus MREDMA approaches. Mercator projection. 


\section{Discussion}

Firstly, the co-ordinate differences shown in Figures 2 through 5 are theoretically invalid offshore Australia in the regions where the transformation parameters have been extrapolated, and thus should not be used. As such, this discussion will only concentrate on the numerical co-ordinate differences on land.

Firstly, the MDMA approach differs from the BWH approach by between $14 \mathrm{~mm}$ and $4.2 \mathrm{~m}$ over continental Australia, and is corroborated by the tests mentioned by Steed (1995). In Figure 2, the co-ordinate differences are smallest near the Johnson station, which forms the origin of the ANS and AGD. If it is assumed for the moment that the BWH approach is exact, then this increased difference on moving away from the Johnson origin could be due to two reasons: The MDMA approach does not account for axial rotations, which explains the direction of the vectors in each quadrant about the Johnson station in Figure 2b. Alternatively, errors in the AGD84 accumulate additively on moving further away from the Johnson origin. However, these errors would also affect the BWH approach, albeit in a different way, thus making the former argument more plausible.

A striking observation in Figures 2 and 3 is the extremely close agreement of the co-ordinate differences derived from the MDMA and TDMA approaches. The difference is virtually indistinguishable, and their numerical values differ by only a few millimetres. This observation has the following simple explanation: The only difference between these approaches is in the use of the flattening and semi-major axes of the respective reference spheroids, which are omitted from the three-parameter model. Therefore, the small difference between these results is to be expected because the values of $\Delta a$ and $\Delta f$ in equations (12) and (13) and equation (11a) are very small, which is indicative of the similar size and shape of the ANS and WGS84 spheroids.

In many other countries, this is not always the case and the effects of the different spheroids can be significant. In Australia however, the co-ordinate transformation from AGD84 to WGS84 using the DMA's origin shift parameters can be performed using the simple three-parameter model with no appreciable loss of accuracy over the standard Molodensky model. The Higgins origin shifts should not be used in the threeparameter model (equation 11b). This is evidenced by the co-ordinate differences shown in Figure 4, which vary between $17.2 \mathrm{~m}$ and $19.8 \mathrm{~m}$ over continental Australia for TH, whereas the differences are between $15 \mathrm{~mm}$ and $4.2 \mathrm{~m}$ for the TDMA approach. The TH approach is theoretically incorrect because the origin shifts for the Bursa-Wolf and Molodensky-Badekas models refer to different origin points, as do the axial rotations. Therefore, the origin shifts are incompatible between the models when used in this way. However, if the threeparameter transformation model is the only option available, the DMA origin shifts should always be used in preference.

In Figure 5, the differences between the BWH and DMAMRE approaches vary by between $12 \mathrm{~mm}$ and $2.4 \mathrm{~m}$ over continental Australia. This difference can reach over 100m offshore Australia, which illustrates the importance of only using projective transformations for interpolation within the area that the transformation parameters were determined. Defense Mapping Agency (1991) state that the multiple regression equations should only be used for mainland Australia. However, based on the comparison in Figure 5, the DMAMRE approach offers a transformation which is compatible at the sub-10m level with the BWH approach in Tasmania. As could have been expected, the multiple regression equation approach is more compatible with the BWH approach than any of the others tested. 


\section{THE ARGUMENTS IN FAVOUR OF PROJECTIVE TRANSFORMATION PARAMETERS}

The differences between the BWH and MREDMA approaches are spatially variant which illustrates the inability of the BWH conformal transformation to model distortions between the AGD84 and WGS84 as determined from satellite surveys conducted in Australia before the mid-1980s. This is also illustrated by the smoother coordinate differences for the conformal models, as is seen by the contours in Figures 2a, 3a and 4a. It is well known that terrestrial geodetic methods are subject to errors which propagate into the co-ordinate datum. These errors are both random and systematic and thus can not accurately modelled by a conformal transformation, especially over the whole continent.

This provides a strong basis for the argument in favour of using spatially varying or projective transformation parameters to transform existing AGD co-ordinate data to the Geocentric Datum of Australia. A projective approach is capable of modelling the differences between the datums and incorporate localised errors in the existing AGD geodetic network in a single operation during the transformation process. After all, as many users and producers of spatial information will probably have to invest the time, effort and incur the expense of changing to this new datum, it should be undertaken as accurately as possible at the time of transition. Moreover, the reliance on a national set of transformation parameters for a conformal transformation is not optimal and localised transformation parameters have already been shown to be superior by Burford (1985), among others. Probably the strongest argument for the use of a projective transformation is to eliminate any errors in the AGD as much as possible instead of simply transforming them, albeit to a lesser extent, directly into the new datum.

The obvious question is how to achieve the most suitable projective transformation. There are already a large number of GPS networks, most notably the Australian Fiducial and National networks (Manning and Harvey, 1994), and their densification networks currently being undertaken by State authorities. The number of GPS networks will inevitably grow before the turn of the century. More importantly, the large proportion of these GPS surveys have been co-located with existing ground points, whose positions are also known in the AGD. Therefore, there are several hundreds or maybe even thousands of points whose co-ordinates are known in both systems so as to allow localised determinations of the transformation parameters with a large degree of redundancy. Therefore, there is no practical or theoretical restriction on the determination and use of projective transformation parameters to transform from the AGD to the GDA.

\section{A proposed approach}

One possible approach for a projective transformation is to determine localised Molodensky-Badekas conformal parameters for a series of sub-networks across the continent in a statistically significant manner using the test proposed by Harvey (1986). These can then be used within the area for which they were determined. However, when using this approach, there may be abrupt discrepancies at the boundaries of these sub-networks.

Therefore, an interpolative approach is proposed by the author, where each of the seven conformal parameters are interpolated between the networks such that they vary as a function of position. An argument against this approach would be the large amount of computer disk space required to store these parameters. However, they could be stored as polynomials (cf. the multiple regression equations) or simply placed on a CDROM together with interface programs that will automatically transform the AGD co-ordinates to the GDA without the need for the user to be aware of the mathematical models or algorithms being used. After all, many of the individuals and organisations who will have to transform data are not necessarily trained in, or even aware of, the intricacies of co-ordinate geodesy. 


\section{CONCLUSIONS AND RECOMMENDATIONS}

It is evident that differences of several metres can occur between existing transformation models and parameters for the transformation from Australian Geodetic Datum 1984 to World Geodetic System 1984, which will have implications for the final implementation of the Geocentric Datum of Australia. This investigation indicates that the Bursa-Wolf/Higgins conformal approach is consistent with the standard Molodensky/DMA conformal approach at the sub-five-metre level over continental Australia. The three-parameter/DMA approach is virtually identical to the standard Molodensky/DMA approach, whilst the three-parameter/Higgins approach should not be used as it introduces a systematic $20 \mathrm{~m}$ co-ordinate error. The difference between the Bursa-Wolf/Higgins and multiple regression equation approaches are less than $2.5 \mathrm{~m}$ and vary irregularly because of the projective nature of the latter approach. This is because the projective transformation can account for localised datum distortions that are effectively invisible, to a large extent, to the conformal model.

This discussion has neglected the accuracy of the parameters themselves, and explains these differences through the actual implementation of the models. Of the methods available in Australia, the Bursa-Wolf model with Higgins's parameters has been considered the most accurate to date. Of equal importance is that it is conceptually simple and straightforward to implement, and has served Australia well over the last few years. However, the projective transformations offer the potential for far more accurate transformations, but this statement can not be verified without a detailed comparison of WGS84 and AGD84 networks, which is beyond the scope of this discussion.

One point of concern is that the Bursa-Wolf model is better suited to the transformation between two satellitederived datums on a global scale. Therefore, the use of this model, although conceptually simple, may not offer the most appropriate choice for Australia, whose datum was established principally by terrestrial measurements. Instead, the Molodensky-Badekas model is better suited to the transformation between satellite and terrestrial geodetic datums. However, the standard Molodensky model in curvilinear co-ordinates as applied by the Defense Mapping Agency is inappropriate as it neglects rotations between the two geodetic datums, which was evidenced by the vector differences from the Bursa-Wolf model. Therefore, of the conformal models available, the most suitable is the Molodensky-Badekas when the rotation matrix is included, together with the co-ordinates of the barycentre (cf. Harvey, 1986; Burford, 1985).

In addition to the conformal transformation models described herein, there are alternative methods which are also suited to the transformation of co-ordinates between satellite and terrestrial geodetic datums. Such conformal models have been proposed by several authors:

- Veis (1960) suggests a model similar to that of Molodensky-Badekas ad Bursa-Wolf where the rotations are applied about a different origin point.

- Hotine (1969) suggests dividing the rotations so as to also account for differences in azimuth, zenith and scale due to distortions in the terrestrial geodetic datum.

- Krakiwsky and Thomson (1974) suggest that the transformation can be determined in two separate stages. Firstly, the origin shift and rotations are determined close to the geodetic origin point, then the remainder of the geodetic network is used to determine further changes in scale and thee-dimensional rotation.

- Wolfrum (1992) proposes a ten parameter model based on an affine transformation where three parameters are used, in addition to those of the Bursa-Wolf or Molodensky-Badekas models, to model distortions in the terrestrial datum by an ellipse.

Therefore, if a conformal model is to be used to transform to the Geocentric Datum of Australia, the most suitable approach should be chosen from all the models currently available and which can deal with distortions in the terrestrial geodetic datum to a certain extent. Despite the existence of these conformal transformation 
models, there is certainly scope for the estimation of spatially varying or projective transformation parameters because of the large number of GPS stations that are now co-located with AGD ground points throughout Australia.

A projective approach can effectively remedy many of the errors in the existing geodetic datum in a single procedure during the transformation process, instead of simply and blindly transforming any errors in the original datum (not necessarily with equal magnitude) to the new datum, as is the case with a conformal transformation. Therefore, it is strongly recommended that projective models and parameters be given serious consideration for transformation to the Geocentric Datum of Australia.

One compromise is to produce both national conformal and projective transformation models and parameters, which can be chosen according to the user's accuracy requirements. However, careful documentation of the method used is essential, especially if it is likely that these data are to be merged in the future after undergoing different transformations that may disagree by several metres.

To conclude, national conformal models, as used at present, are unable to accurately account for most of the errors existing between satellite and terrestrial geodetic datums. Therefore, a projective transformation is recommended instead, such as the multiple regression equation or interpolation of Molodensky-Badekas parameters between sub-networks. Such projective methods will transform the AGD co-ordinates according to the change in datum, whilst accounting for any errors existing in the AGD which have been identified by the more precise GPS surveys. Most importantly, a projective transformation will allow a more direct and rigorous transformation for those States that still use AGD66, instead of the involved process of staging co-ordinates via the AGD84 (usually using a two-dimensional conformal transformation).

\section{ACKNOWLEDGEMENTS}

This paper was written whilst the author was a Visiting Professor at the Department of Geodesy and Geomatics Engineering, University of New Brunswick, Canada. The hospitality of Professor Petr Vanicek and his colleagues is therefore gratefully acknowledged, as is financial assistance from the author's employer, Curtin University of Technology. The figures were produced using the algorithms contained within the public domain software of Wessel and Smith (1995) [New version of the Generic Mapping Tools released. EOS - Transactions of the American Geophysical Union, vol. 72, no. 441, pp. 445-446], which is available via FTP over the Internet. I would also like to thank the four anonymous reviewers for their constructive suggestions.

\section{REFERENCES}

Applebaum, L.T. (1982) Geodetic datum transformation by multiple regression equations. Proceedings of the Third International Geodetic Symposium on Satellite Doppler Positioning, Las Cruces, New Mexico, pp. 207-223.

Badekas, J. (1969) Investigations related to the establishment of a world geodetic system, Report 124, Department of Geodetic Science, Ohio State University, Columbus.

Borkowski, K.M. (1989) Accurate algorithms to transform geocentric to geodetic co-ordinates, Bulletin Géodésique, vol. 63, pp. 50-56.

Bowring, B.R. (1985) The accuracy of geodetic latitude and height equations, Survey Review, vol. 28, no. 218, pp. 202-206.

Burford, B.J. (1985) A further examination of datum transformation parameters in Australia, The Australian Surveyor, vol. 32, no. 7, pp. 536-558. 
Bursa, M. (1962) The theory for the determination of the non-parallelism of the minor axis of the reference ellipsoid and the inertial polar axis of the Earth, and the planes of the initial astronomic and geodetic meridians from observations of artificial Earth satellites, Studia Geophysica et Geodetica, no. 6, pp. 209-214.

Defense Mapping Agency (1991) Department of Defense World Geodetic System 1984: its definition and relationships with local geodetic systems (second edition). Technical Report no. 8350.2, Defense Mapping Agency, Washington

Featherstone, W.E. (1994) An explanation of the Geocentric Datum of Australia and its effects upon future mapping, Cartography, vol. 23, no. 2, pp. 1-12 and vol. 24, no. 1, pp. 43-44.

Featherstone, W.E. (1996) A revised explanation of the Geocentric Datum of Australia and its effects upon future mapping, The Australian Surveyor, vol. 41, no. 2, pp. 121-130.

Harvey, B.R. (1986) Transformation of 3D co-ordinates. The Australian Surveyor, vol. 33, no. 2, pp. 105-125.

Higgins, M.B. (1987) Transformation from WGS84 to AGD84, an interim solution, Internal Report, Department of Geographic Information, The University of Queensland.

Higgins, M.B. (1994) The effect on mapping sciences of adoption of a geocentric datum by the year 2000, Proceedings of Mapping Sciences 94, Gold Coast, September, pp. 161-168.

Hotine, M. (1969) Mathematical Geodesy, ESSA Monograph no. 2, United States Department of Commerce, Washington.

Inter-governmental Committee on Surveying and Mapping (1994) A new era for Australia. Information Circular, Inter-governmental Committee on Surveying and Mapping, Belconnen.

Krakiwsky, E.J. and Thomson, D.B. (1974) Mathematical models for the combination of terrestrial and satellite networks, The Canadian Surveyor, vol. 28, no. 5, pp. 606-615.

Manning, J. and Harvey, W.M. (1994) Status of the Australian geocentric datum. The Australian Surveyor, vol. 39, no. 1, pp. 28-33.

Molodensky M.S., Eremeev V.F. and Yurkina M.I. (1962) Methods for Study of the External Gravitational Field and Figure of the Earth. Israeli Programme for the Translation of Scientific Publications, Jerusalem.

Paul, M.K. (1973) A note on computation of geodetic co-ordinates from geocentric (Cartesian) co-ordinates. Bulletin Géodésique, no. 108, pp. 135-139.

Soler, T. (1976) On differential transformations between Cartesian and curvilinear co-ordinates. Report 236, Department of Geodetic Science, Ohio State University, Columbus.

Steed, J. (1995) The geocentric datum of Australia. Surveying World, vol. 4, no. 1, pp. 14-17.

Vanicek, P. and Krakiwsky, E.J. (1986) Geodesy: The Concepts (second edition), North-Holland, Amsterdam.

Veis, G. (1960) Geodetic uses of artificial Earth satellites. Smithsonian Contributions to Astrophysics, vol. 3, no. 9, pp. 95-161.

Wolf, H. (1963) Geometric connection and re-orientation of three-dimensional triangulation nets. Bulletin Géodésique, no. 68, pp. 165-169.

Wolfrum, O. (1992) Merging terrestrial and satellite networks by a ten-parameter transformation model. Manuscripta Geodaetica, vol. 17, no. 4, pp. 210-214. 